Research Article

\title{
Integrated Inductor Compact Modeling Methodology
}

\author{
Y. Moisiadis *, S. Stefanou and P. Papadopoulos \\ Helic S.A., 12 Sorou str., Maroussi 15125, Athens, Greece
}

Received 30 June 2015; Accepted 15 January 2016

\begin{abstract}
In this paper a methodology will be presented for compact modeling of integrated inductors. The compact model is capable of capturing all modes of operation (single-ended, differential) for inductors with or without center tap. For such purpose an enhanced compact circuit is utilized, while a specific fitting methodology is introduced, based on the detailed model S-parameters.
\end{abstract}

Keywords: Integrated inductor, Compact model, RFIC

\section{Introduction}

Integrated inductors are key components for radio frequency (RF) circuits, such as VCO, LNA and PA, since they determine the overall performance in terms of noise, gain, power dissipation. Modeling integrated inductors, especially for mm-wave applications, has become a challenging task due to the plethora of effects that must be taken into account (skin, proximity, substrate coupling, e.t.c). The requirement of increased accuracy in conjunction with the large number of inductors that a circuit may include, significantly increases the number of elements of the model (RLCk netlist) extracted from an Electromagnetic (EM) tool.

Consequently, a compact inductor model is indispensable to ensure fast and accurate simulations, reducing the design and verification cycle-time. To bring most of the benefits, a compact inductor model must precisely replicate the full model behaviour at least up to the first self-resonance frequency. Furthermore, to increase IC design flexibility the compact model must accurately operate independent of the excitation mode (single-ended or differential) or the connection of the center tap (connected or floating) [1]. In addition, integrating the compact modeling methodology into a typical IC design flow requires that the compact modeling procedure imposes not significant memory and time overhead, while it should be based on reference data that can be easily extracted from the full model.

The proposed compact model (CM) methodology is based on the double- $\pi$ inductor model and adopts a specific constrained optimization procedure to evaluate the parameters of the components of the CM. The methodology requires no prior knowledge of the technology parameters or the geometry of the inductor.

\section{Inductor Compact Model}

The compact circuit, illustrated in Fig.1, is based on the

\footnotetext{
*E-mail address: y. moisiadis@helic.com

ISSN: $1791-2377$ @ 2016 Eastern Macedonia and Thrace Institute of Technology. All rights reserved.
}

widely used double- $\pi$ model configuration $[2,3]$, with some additional elements to increase accuracy. A series $\mathrm{R}$ and $\mathrm{L}$ is used in each branch of the model (Rs1, Ls1 and Rs2, Ls2 respectively), while a three ladder network (instead of one) is utilized in each branch (R1, R1a, R1b, L1, L1a, L1b) (R2, R2a, R2b, L2, L2a, L2b) to accurately model the skin and proximity effect. Coxj represents the capacitance between the conductor and the silicon substrate, while Csubj and Rsubj are the substrate capacitance and resistance respectively. Csoj, Rsoj are inserted to realize the lateral coupling among the metal paths. The three main inductors Ls1, Ls2, Lct exhibit mutual coupling between them.

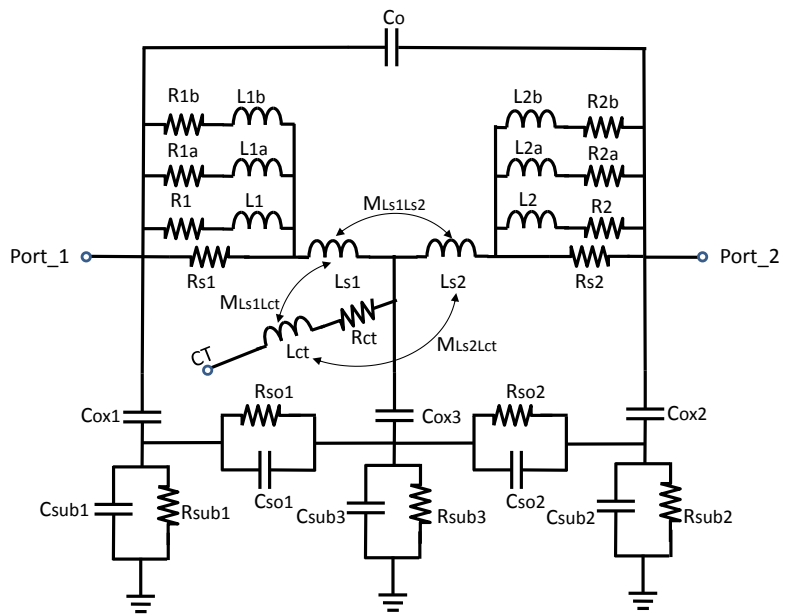

Fig. 1. Inductor Compact Model

The elements of the compact model can be calculated either directly from the geometry of the inductor, applying an analytical model extraction method $[3,4]$ or from the Sparameters using a set of closed equations $[5,6]$. However, both methodologies are of limited accuracy and include several parameters which are process related.

\section{Compact Model Extraction Methodology}

The proposed approach utilizes on a non-linear constrained optimization procedure based on the detailed model Sparameters. For the case of inductor without center tap, in order 07 
k), a two-step non-linear constrained optimization procedure is implemented. The procedure is based on Trust-Region (TR) optimization algorithm as implemented in MKL mathematical library. Initially, physical ranges of each parameter value are defined and used as constraints for the optimization procedure. As initial guess for the solution, the central point of each range is defined.

For inductors with no center tap the optimization procedure is performed in two steps: During the first one, the objective function is the percentage deviation of the $\mathrm{Y}$ parameters between the detailed and the compact model, sampled over a frequency range up to the first self-resonance frequency. In the second step, using as initial guess the parameters obtained from the first step, a new objective function is used calculating for the single-ended and differential operation, the percentage deviations of specific metrics, such as: inductance and resistance at $100 \mathrm{MHz}(\mathrm{Ldc} \& \mathrm{Rdc})$, maximum quality factor (Qmax) and self-resonance frequency.

For inductors with center tap an additional initial step in the optimization procedure is added. First, an optimization is performed using a floating center tap model, matching the $\mathrm{Y}$ parameters between the detailed and the compact model in a similar way as in the first step of the case of the inductor without center tap. During this step, the parameters Lct, Rct and MLs1Lct, MLs2Let have no contribution. Then, based on the parameter values obtained from the first optimization, a twostep optimization procedure is followed where a combined objective function is adopted. The parameters of the compact model are evaluated to simultaneously reproduce the performance of the inductor for the two modes of operation (center tap grounded and floating). In this way, a parameter optimization minimizing Y-parameter error in the first step and Ldc, Rdc, Qmax and self-resonance frequency in the second step for both modes of operation is obtained. Fig. 2 presents the main algorithmic steps of the compact model flow.

For single-ended operation the main metrics of the inductor used in the optimization flow are the inductance (Lse) the quality factor (Qse) and the resistance (Rse), calculated from the Y-parameters based on the following equations:

$L_{s e}=\frac{\operatorname{imag}\left(\frac{1}{Y 11}\right)}{2 \pi f}$

$Q_{\text {se }}=\frac{\operatorname{imag}\left(\frac{1}{Y 11}\right)}{\operatorname{real}\left(\frac{1}{Y 11}\right)}$

$R_{s e}=\operatorname{real}\left(\frac{1}{Y 11}\right)$

For differential operation, the inductance (Lse), quality factor (Qse) and resistance (Rse), can be efficiently calculated from the S-parameters, as shown in the equations below:

$$
\begin{aligned}
& S_{\text {diff }}=\frac{S 11+S 22-S 12-S 21}{2} \\
& Z_{\text {diff }}=100 * \frac{1+S_{\text {diff }}}{1-S_{\text {diff }}} \\
& L_{\text {diff }}=\frac{\operatorname{imag}\left(Z_{\text {diff }}\right)}{2 \pi f} \\
& Q_{\text {se }}=\frac{\operatorname{imag}\left(Z_{\text {diff }}\right)}{\operatorname{real}\left(Z_{\text {diff }}\right)} \\
& R_{\text {diff }}=\operatorname{real}\left(Z_{\text {diff }}\right)
\end{aligned}
$$

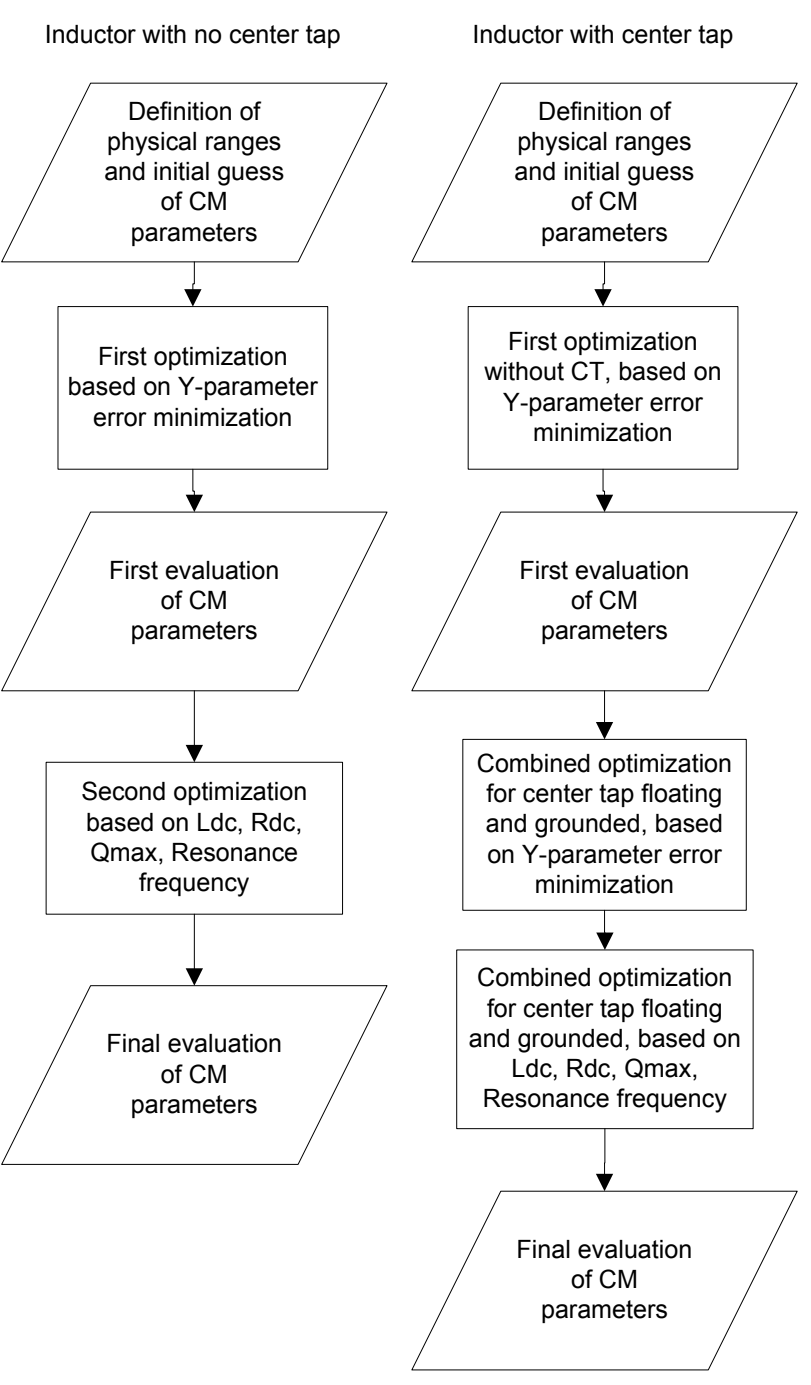

Fig. 2. Compact model algorithmic flow

\section{Simulation Results}

Fig. 3 and 4 present the L, Q and R for an inductor with no center tap, for single-ended and differential mode respectively. Fig. 5 and 6 illustrate the L, Q and R for an inductor with center tap floating (single-ended and differential mode respectively), while Fig. 7 shows the results for the same inductor with the center-tap connected to ground. The performance metrics (L, Q, R) have been simulated from the original and compact model and exhibit very good match up to the self-resonance frequency.

The compact model extraction methodology has been validated for a set of 100 of inductors in different processes, exhibiting an inductance range from $0.5 \mathrm{nH}$ to $5 \mathrm{nH}$ and selfresonance frequency range from $10 \mathrm{GHz}$ to $50 \mathrm{GHz}$. Simulation results for the full set of inductors verified that up to the first resonance frequency, the compact model successfully replicates the performance characteristics of the original model (inductance, resistance, quality factor), introducing a maximum error of $7 \%$. The maximum time required to synthesize the compact model was $7 \mathrm{~m}$, when running the algorithm in a QuadCore processor with 16GB RAM. 


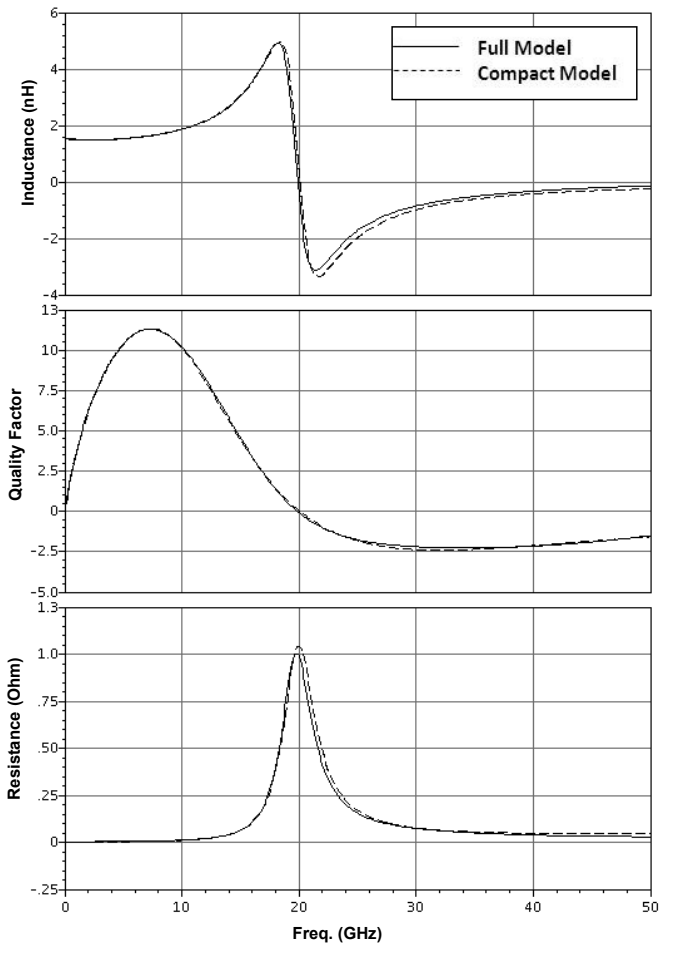

Fig. 3. L, Q and R for inductor with no center tap (Single-Ended Mode)

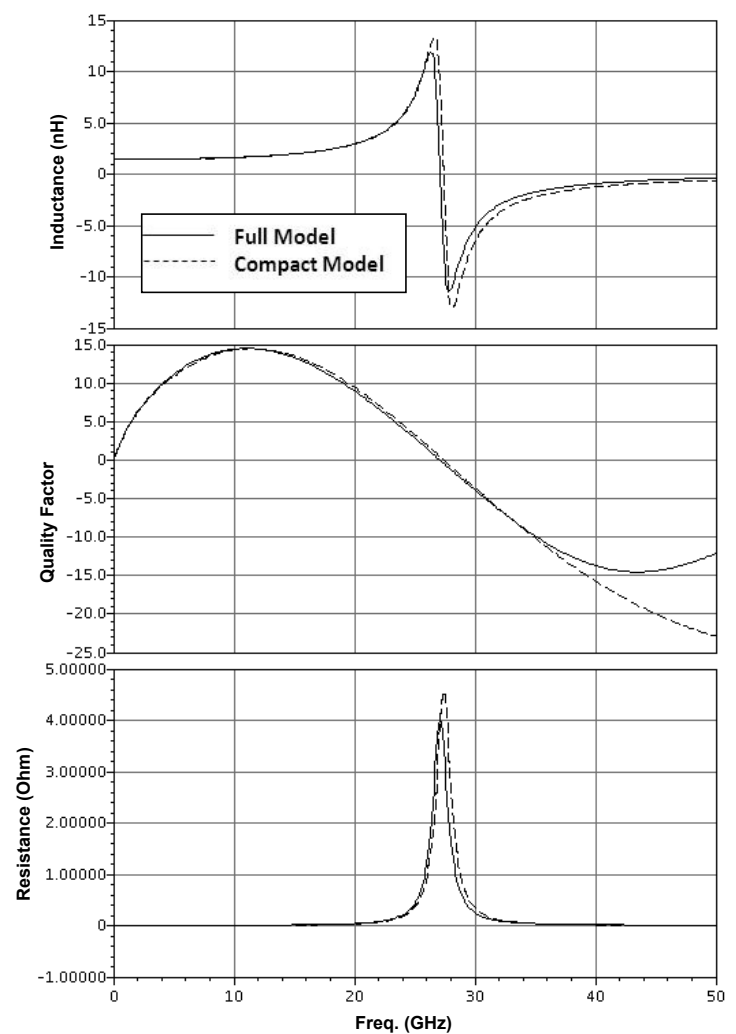

Fig. 4. L, Q and R for inductor with no center tap (Differential Mode)

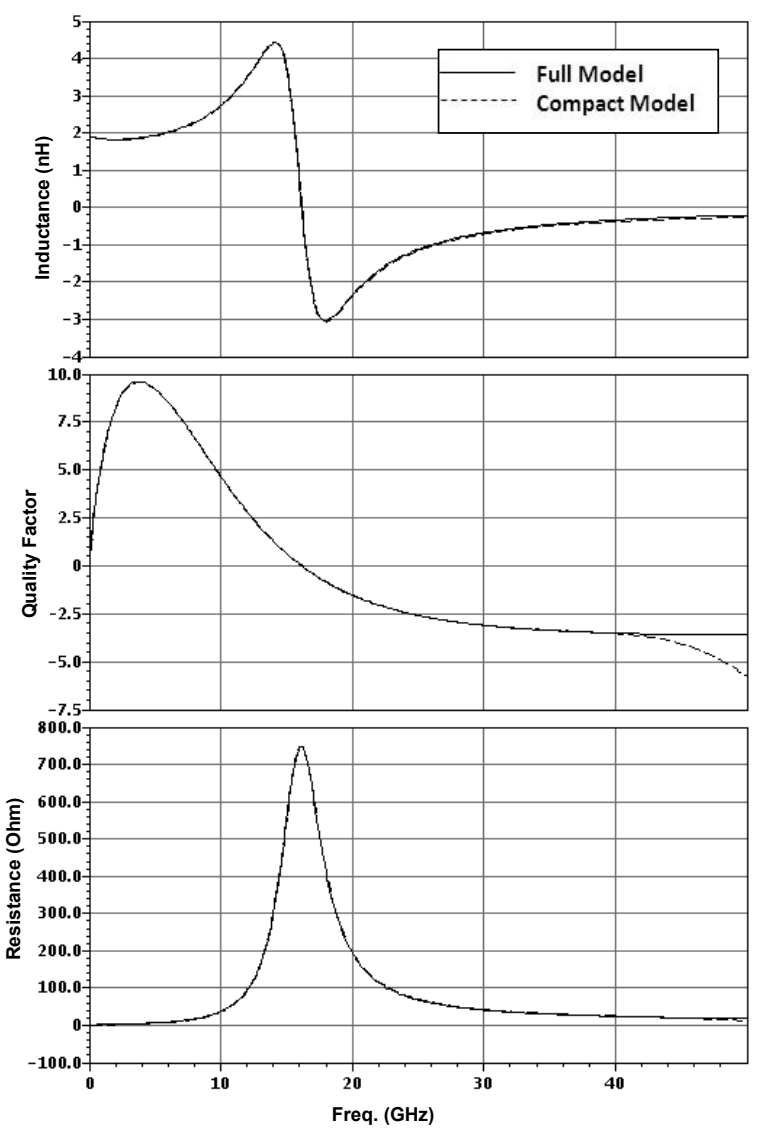

Fig. 5. L, Q and R for inductor with center tap floating (Single-Ended Mode)

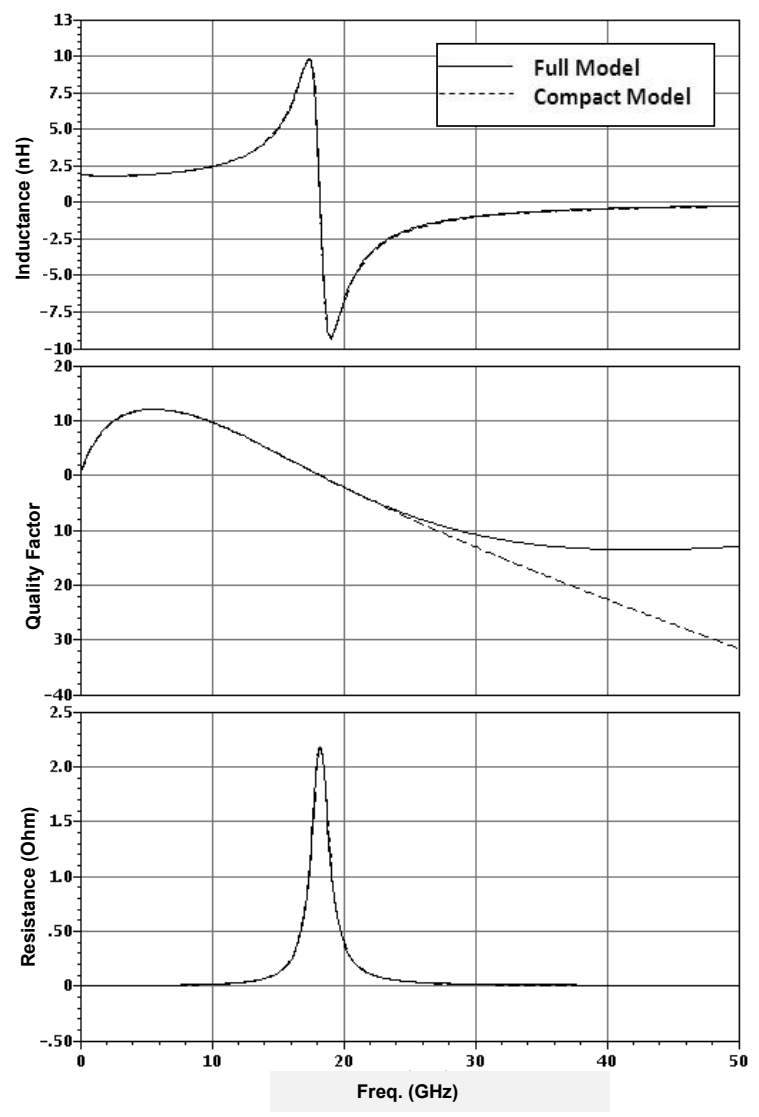

Fig. 6. L, Q and $\mathrm{R}$ for inductor with center tap floating (Differential Mode) 


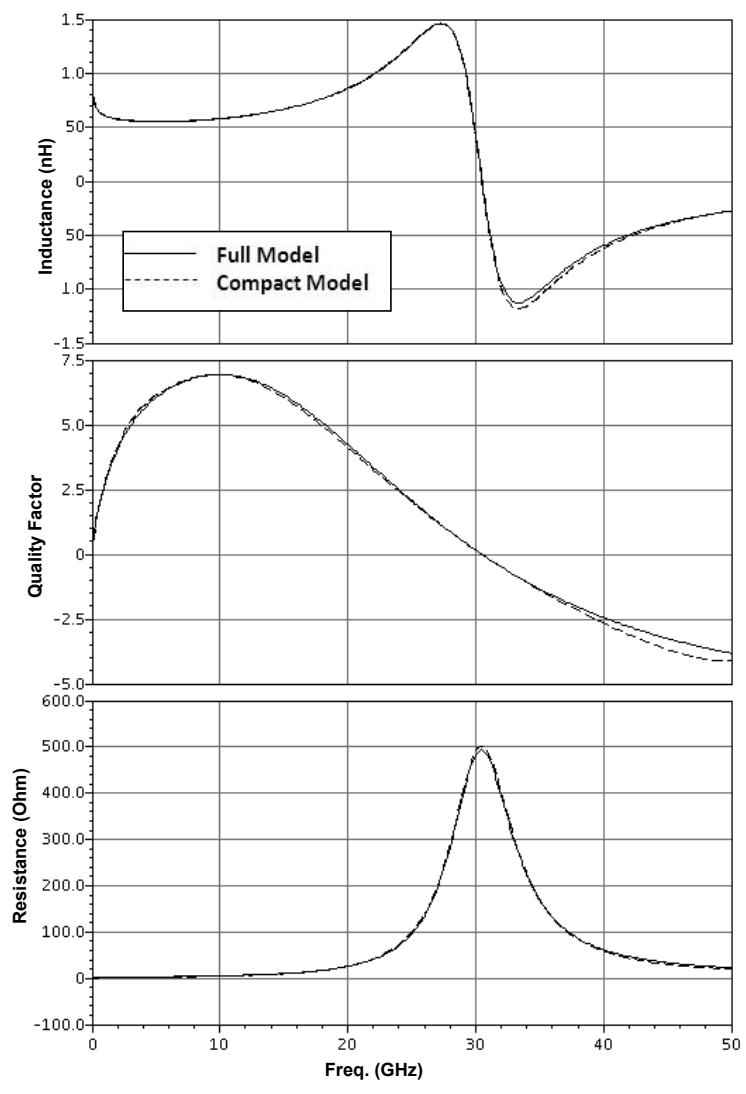

Fig. 7. L, Q and R for inductor with center tap grounded (Single-Ended Mode)
This paper was presented at Pan-Hellenic Conference on Electronics and Telecommunications - PACET, that took place May 8-9 2015, at Ioannina Greece.

\section{Conclusions}

A methodology is proposed for the synthesis of a compact model for integrated inductors. The compact model is based on the double- $\pi$ inductor model and utilizes a fitting methodology realized with a constrained optimization procedure to evaluate the elements of the equivalent circuit. The compact model is capable of capturing all modes of operation (single-ended, differential) for inductors with and without center tap. The optimization is based on the detailed model S-parameters and it is independent of the technology parameters or the geometry of the inductor.

The compact model synthesized form the specific methodology successfully replicated the performance of a set of 100 inductors, introducing a maximum error of $7 \%$ for the main performance metrics.

\section{References}

1. Okada, K., Sugawara, H., Nishikawa Kazuyasu, Masu, K, "Modeling of three-pPort $\mathrm{Si}$ CMOS on-chip symmetric inductor for various operation modes", European Microwave Conference, pp. 520-523, 2007

2. Fengyi (Fred) Huang, Jingxue Lu, Nan Jiang, Xiaowen Zhang, Wengang $\mathrm{Wu}$, and Yangyuan Wang. "Frequency-Independent Asymmetric Double- $\pi$ Equivalent Circuit for On-Chip Spiral Inductors: Physics-Based Modeling and Parameter Extraction", IEEE Journal of Solid State Circuits, vol. 41, No. 10, pp. 419-426, Oct. 2006

3. Young-Ghyu Ahn, Seong-Kyun Kim, Jung-Hoon Chun, Byung-Sung Kim, "Efficient Scalable Modeling of Double- $\pi$ Equivalent Circuit for On-Chip Spiral Inductors", IEEE Transactions on Microwave Theory and Techniques, $2289-2300$, vol. 57, No. 10, pp. $2289-2300$, Aug. 2009

4. Wei Gao, Zhiping Yu, "Scalable compact circuit model and synthesis for RF CMOS spiral inductors", IEEE Transactions on Microwave Theory and Techniques, vol. 54 , Issue: 3 , pp. $1055-1064$. March 2006

5. Myounggon Kang, Gil, J., Hyungcheol Shin, "A simple parameter extraction method of spiral on-chip inductors", IEEE Transactions on Electron Devices, vol. 52 , Issue: 9, pp. 1976 - 1981, Sept. 2005

6. Hao-Hui Chen, Huai-Wen Zhang, Shyh-Jong Chung, Jen-Tsai Kuo, Tzung-Chi Wu, "Accurate Systematic Model-Parameter Extraction for On-Chip Spiral Inductors", IEEE Transactions on Electron Devices, vol. 55 , Issue: 11, pp. 3267 - 3273, Nov. 2008 